Draft Version November 3, 2018

Preprint typeset using LTEX style emulateapj v. 11/10/09

\title{
TEMPORAL PROPERTIES OF GX 301-2 OVER A YEAR-LONG OBSERVATION WITH SUPERAGILE
}

\author{
Y. Evangelista ${ }^{1,2, *}$, M. Feroci ${ }^{1}$, E. Costa ${ }^{1}$, E. Del Monte $^{1}$, I. Donnarumma ${ }^{1}$, I. Lapshov ${ }^{1}$, F. Lazzarotto ${ }^{1}$, L. Pacciani $^{1}$, M. Rapisarda $^{3}$, \\ P. Soffitta ${ }^{1}$, A. Argan ${ }^{1}$, G. Barbiellini ${ }^{4,5}$, F. Boffelli ${ }^{6}$, A. Bulgarelli ${ }^{7}$, P. Caraveo ${ }^{8}$, P.W. Cattaneo ${ }^{6}$, A. Chen ${ }^{8}$, F. D’ Ammando ${ }^{1,9}$,

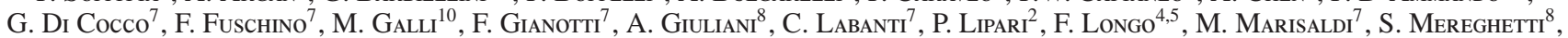

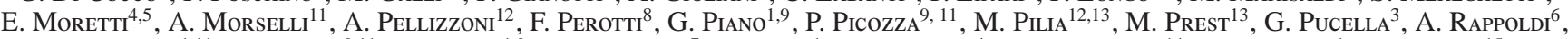 \\ S. Sabatini ${ }^{1,11}$, E. Striani ${ }^{9,11}$, M. Tavani ${ }^{1,9}$, M. Trifoglio ${ }^{7}$, A. Trois ${ }^{1}$, E. Vallazza ${ }^{4}$, S. Vercellone ${ }^{14}$, V. Vittorini ${ }^{1}$, A. Zambra $^{15}$, \\ L. A. Antonelli ${ }^{16,17}$, S. Cutini ${ }^{16,18}$, C. Pittori ${ }^{16,18}$, B. Preger ${ }^{16,18}$, P. Santolamazza ${ }^{16,18}$, F. Verrecchia ${ }^{16,18}$, P. Giommi $^{16,19}$, L. Salotti ${ }^{19}$ \\ Draft version November 3, 2018
}

\begin{abstract}
We present the long-term monitoring of the High Mass X-ray Binary GX 301-2 performed with the SuperAGILE instrument on-board the AGILE mission. The source was monitored in the $20-60 \mathrm{keV}$ energy band during the first year of the mission from 2007 July 17 to 2008 August 31, covering about one whole orbital period and three more pre-periastron passages for a total net observation time of about 3.7 Ms. The SuperAGILE dataset represents one of the most continuous and complete monitoring at hard X-ray energies of the 41.5 day long binary period available to date.

The source behavior was characterized at all orbital phases in terms of hard X-ray flux, spectral hardness, spin period history, pulsed fraction and pulse shape profile. We also complemented the SuperAGILE observations with the soft X-ray data of the RossiXTE/ASM. Our analysis shows a clear orbital modulation of the spectral hardness, with peaks in correspondence with the pre-periastron flare and near phase 0.25 . The hardness peaks we found could be related with the wind-plus-stream accretion model proposed in order to explain the orbital light curve modulation of GX 301-2.

Timing analysis of the pulsar spin period shows that the secular trend of the $\sim 680 \mathrm{~s}$ pulse period is consistent with the previous observations, although there is evidence of a slight decrease in the spin-down rate. The analysis of the hard X-ray pulsed emission also showed a variable pulse shape profile as a function of the orbital phase, with substructures detected near the passage at the periastron, and a clear modulation of the pulsed fraction, which appears in turn strongly anti-correlated with the source intensity.

Subject headings: pulsars: individual: GX 3012 stars: neutron X-rays: binaries
\end{abstract}

\section{INTRODUCTION}

GX 301-2 is a High Mass X-ray Binary (HMXB) system containing an $\mathrm{X}$-ray pulsar, with a $\sim 685 \mathrm{~s}$ rotation pe-

${ }^{1}$ INAF IASF Roma, Via Fosso del Cavaliere 100, I-00133 Roma, Italy

${ }^{2}$ Dip. di Fisica, Università degli Studi di Roma "La Sapienza", P.le A. Moro 5, I-00185 Roma, Italy

${ }^{3}$ ENEA, Via E. Fermi 45, I-00044 Frascati (Rm), Italy

${ }^{4}$ INFN Trieste, Padriciano 99, I-34012 Trieste, Italy

${ }^{5}$ Dip. di Fisica, Università di Trieste, Via Valerio 2, I-34127 Trieste, Italy

${ }^{6}$ INFN Pavia, Via Bassi, 6 I-27100 Pavia, Italy

${ }^{7}$ INAF IASF Bologna, Via Gobetti 101, I-40129 Bologna, Italy

${ }^{8}$ INAF IASF Milano, Via E. Bassini 15, I-20133 Milano, Italy

${ }^{9}$ Dip. di Fisica, Università degli Studi di Roma "Tor Vergata", Via della Ricerca Scientifica 1, I-00133 Roma, Italy

${ }^{10}$ ENEA C.R. "E. Clementel", Via don Fiammelli 2, I-40128 Bologna, Italy

${ }^{11}$ INFN Roma Tor Vergata, Via della Ricerca Scientifica 1, I-00133, Roma, Italy

12 INAF Osservatorio Astronomico di Cagliari, loc. Poggio dei Pini, strada 54, I-09012, Capoterra (Ca), Italy

${ }^{13}$ Dip. di Fisica e Matematica, Università dell'Insubria, Via Valleggio 11, I-20100 Como, Italy

${ }^{14}$ INAF IASF Palermo, Via U. La Malfa 153, I-90146 Palermo, Italy

${ }^{15}$ Consorzio Interuniversitario per la Fisica Spaziale, Viale Settimio Severo 63, I-10133 Torino, Italy

${ }^{16}$ ASI Science Data Center, Via G. Galilei, I-00044 Frascati (Rm), Italy

${ }^{17}$ INAF Osservatorio Astronomico di Roma, Via di Frascati 33, I-00040 Monte Porzio Catone (Rm), Italy

${ }^{18}$ INAF personnel resident at ASI Science Data Center

${ }^{19}$ Agenzia Spaziale Italiana, Unità Osservazione dell'Universo, Viale Liegi 26, 00198 Roma, Italy

yuri.evangelista@iasf-roma.inaf.it riod, orbiting the B-emission line hypergiant star $(\mathrm{B} 1 \mathrm{Ia}+)$ Wray 977. The X-ray source was discovered in 1969 April (Lewin et al. 1971) with a balloon observation, subsequently White et al. (1976) discovered the $700 \mathrm{~s}$ X-ray pulsation. The pulsar orbit has an eccentricity of 0.462 (Koh et al. 1997) and a period of $\sim 41.5$ days (White et al. 1978; Sato et al. 1986; Koh et al. 1997; Doroshenko et al. 2008). The mass function of the system is $31.1 \mathrm{M}_{\odot}$ with a companion mass in the range $39<\mathrm{M}<53 \mathrm{M}_{\odot}$, estimated by the measurement of optical radial velocity amplitude (Kaper et al. 2006). The radius of Wray 977 obtained by Kaper et al. (2006) by fitting atmosphere model is $62 R_{\odot}$, while the effective temperature is about $1.8 \times 10^{4} \mathrm{~K}$.

GX 301-2 shows regular X-ray flares about two days before the periastron passage, while a secondary intensity peak was sometimes observed near the apastron passage (Pravdo et al. 1995; Koh et al. 1997; Pravdo \& Ghosh 2001). Assuming a distance of $3 \mathrm{kpc}$, as derived by Kaper et al. (2006), the source luminosity during pre-periastron (PP) flares reaches values of $\sim 10^{37} \mathrm{erg} \mathrm{s}^{-1}$, about 25 times larger than the luminosity showed by the source in the other orbital phases.

Since its discovery, GX 301-2 exhibited unpredictable torque variations superimposed on a secular spin trend, which passed from a spin-up to a spin-down state between MJD 48500 and MJD 49500. Two rapid spin-up episodes with $\dot{v} \sim 2-5 \times 10^{-12} \mathrm{~Hz} \mathrm{~s}^{-1}$ were also found by Koh et al. (1997) analyzing BATSE data, thus confirming the erratic behavior of the pulsar spin period. Koh et al. (1997) suggested that the long-term spin-up trend observed between 1984 and 1994 might not be regular and continuous, but due to sev- 
eral rapid spin-up episodes, which in turn could be related to the formation of a temporary accretion disk. Pravdo \& Ghosh (2001), using BATSE data, found evidence that the hard X-ray (20-50 keV) intensity of the binary system during quiescent orbital phases is correlated with the pulse period, suggesting a possible relation between the spin period behavior and the mass accretion rate.

Several wind accretion models were proposed to explain both the regular PP and apastron flaring activities of the source. A dense disk around Wray 977, which intercepts the neutron star (NS) orbit near the periastron and the apastron, was proposed by Pravdo et al. (1995) and Pravdo \& Ghosh (2001). However, this model seems unable to provide a satisfactory modeling of the observed orbital X-ray light curve of the source (Leahy 2002) and optical observations by Kaper et al. (2006) found no confirmation of the presence of a disc. The existence of a high density stream of matter in addition to the stellar wind was proposed by Haberl (1991); Leahy (1991, 2002); Leahy \& Kostka (2008). The stream outflows from Wray 977 due to tidal interaction intercepts the NS orbit near the periastron and the apastron, causing a large increase in the mass accretion rate with a sudden enhancement of the X-ray luminosity from the neutron star. Signatures of the presence of such a stream were found by Saraswat et al. (1996) analyzing the low-energy excess in the ASCA spectrum of GX 301-2, and also by Kaper et al. (2006) from the orbital modulation of the spectral lines detected in the stellar wind of the optical companion. The wind-plus-stream model predicts also a large variation in the column density as a function of the orbital phase. The $N_{\mathrm{H}}$ is expected to change from a value of $\sim 10^{23} \mathrm{~cm}^{-2}$ at orbital phase 0.8 to values greater than $10^{24} \mathrm{~cm}^{-2}$ during the PP flares and between orbital phases $0.2-0.3$.

We observed the HMXB GX 301-2 with the SuperAGILE (SA) experiment onboard the Astro-rivelatore Gamma ad Immagini LEggero (AGILE) satellite during the first year of the mission. AGILE, launched on 2007 April 23, is a scientific mission of the Italian Space Agency dedicated to high-energy astrophysics (Tavani et al.2009). In the following sections we report on the analysis of the SuperAGILE data, in the energy range 20-60 keV. In Section 2 we describe the SuperAGILE experiment and the observations of GX 301-2 we performed, in Section 3 we present the timing analysis of the pulsar emission and in Section 4 we analyze the orbital light curve of GX 301-2 using the hard X-ray SuperAGILE and the soft Xray $R X T E / A S M$ data and the orbital variability of the source pulsed fraction. Discussion of the data analysis and the interpretation of the results are presented in Section 5, while in Section 6 we draw our conclusions.

\section{SUPERAGILE OBSERVATIONS AND DATA REDUCTION}

\subsection{The SuperAGILE Experiment}

SuperAGILE (see Feroci et al. 2007 for an extensive description of the experiment) is the hard X-ray monitor of the AGILE mission and is based on four one-dimensional codedmask instruments, each one equipped with $\mathrm{a} \sim 360 \mathrm{~cm}^{2}$ silicon microstrip detector and a tungsten mask-collimator system. The experiment has $\sim 1 \mathrm{sr}$ field of view (FoV), 6 arcmin angular resolution, and a point source location accuracy better than 2 arcmin for intense sources. SuperAGILE is able to detect X-ray photons between $18 \mathrm{keV}$ and $60 \mathrm{keV}$ with a resolution of $\sim 8 \mathrm{keV}$ FWHM and an on-axis effective area of $250 \mathrm{~cm}^{2}$. The time resolution of the instrument is $2 \mu \mathrm{s}$ while the accuracy is about $5 \mu \mathrm{s}$. The dead time is negligible in normal operation conditions, except for the passages through the South Atlantic Geomagnetic Anomaly where the vetoes from the AGILE anticoincidence systems reduce the SA live time to $\sim 10 \%$ or less. In the standard analysis this fraction of the orbit is usually removed from the data.

In standard operation mode SuperAGILE provides event by event data, from which position and normalized count rates for each source detected in the FoV are derived. The data are downloaded from the spacecraft about every 100 minutes during the AGILE passage over the Malindi ground station, sent to the ASI Science Data Center (ASDC) in Rome and then processed by the SuperAGILE Scientific Pipeline (SASOA) developed by the SuperAGILE team at INAF/IASF Rome (Lazzarotto et al. 2008) and running both at the ASDC and at IASF. The automated analysis of the SuperAGILE data autonomously updates a source catalog and orbital light curves for bright sources are then posted at the ASDC web site 21 .

\subsection{SuperAGILE Observations of GX 301-2}

SuperAGILE observations of the HMXB GX 301-2 started in mid 2007 July, just after the end of the AGILE Commissioning Phase, as part of the Science Verification Phase. Indeed, GX 301-2 was the first light of the experiment. Due to the solar constraints and the AGILE pointing strategy the satellite is operated by performing long observations, typically of 2 4 weeks duration, during which the pointing direction slowly drifts (at a rate of $\sim 1^{\circ}$ day $^{-1}$ ). SuperAGILE performed six long observations of GX 301-2, for a total net exposure time of about $3.7 \times 10^{6} \mathrm{~s}$. Table 1 shows the journal of the observations. The first observation and part of the second one were performed during the AGILE Science Verification Phase that started in mid 2007 July and ended on 2007 November 30. The other observations were carried out during the AGILE AO1 program, which started on 2007 December 1 and lasted one year. The last column in Table 1 shows the source offaxis angle in the SuperAGILE FoV for each observation. The configuration of SuperAGILE implies that the source counts collected by the instrument strongly depend on the off-axis angle (see e.g. Feroci et al. 2007), and thus also the detection significance is dependent on the source position in the FoV.

The SuperAGILE observations took place in different segments of the GX 301-2 binary orbit. In Figure 1 we graphically show the orbital phases of our six observations: three of them covered a large fraction of a complete binary orbit (Observations ID II, III and V), while the other three were shorter, and covered the equivalent of one full binary orbit all together, but arranged over three different orbits. The SuperAGILE data thus allow for a often continuous monitoring of the complete 41.5 days binary cycle. It is worth noting that the monitoring of four different passages at the pre-periastron orbital phase (0.95) was included in the SuperAGILE observations.

\subsection{Data Reduction and Analysis}

SuperAGILE data reduction was performed using the standard SASOA pipeline (Lazzarotto et al. 2008), which extracts high level products from the photon-by-photon SuperAGILE data. Production of source images was performed with the SuperAGILE Enhanced Multi Imaging (EMI) procedure

\footnotetext{
${ }^{21} \mathrm{http}: / /$ agile.asdc.asi.it/sagilecat_sources.htm

$22 \mathrm{http}$ ///agile.asdc.asi.it/current_pointing.html
} 

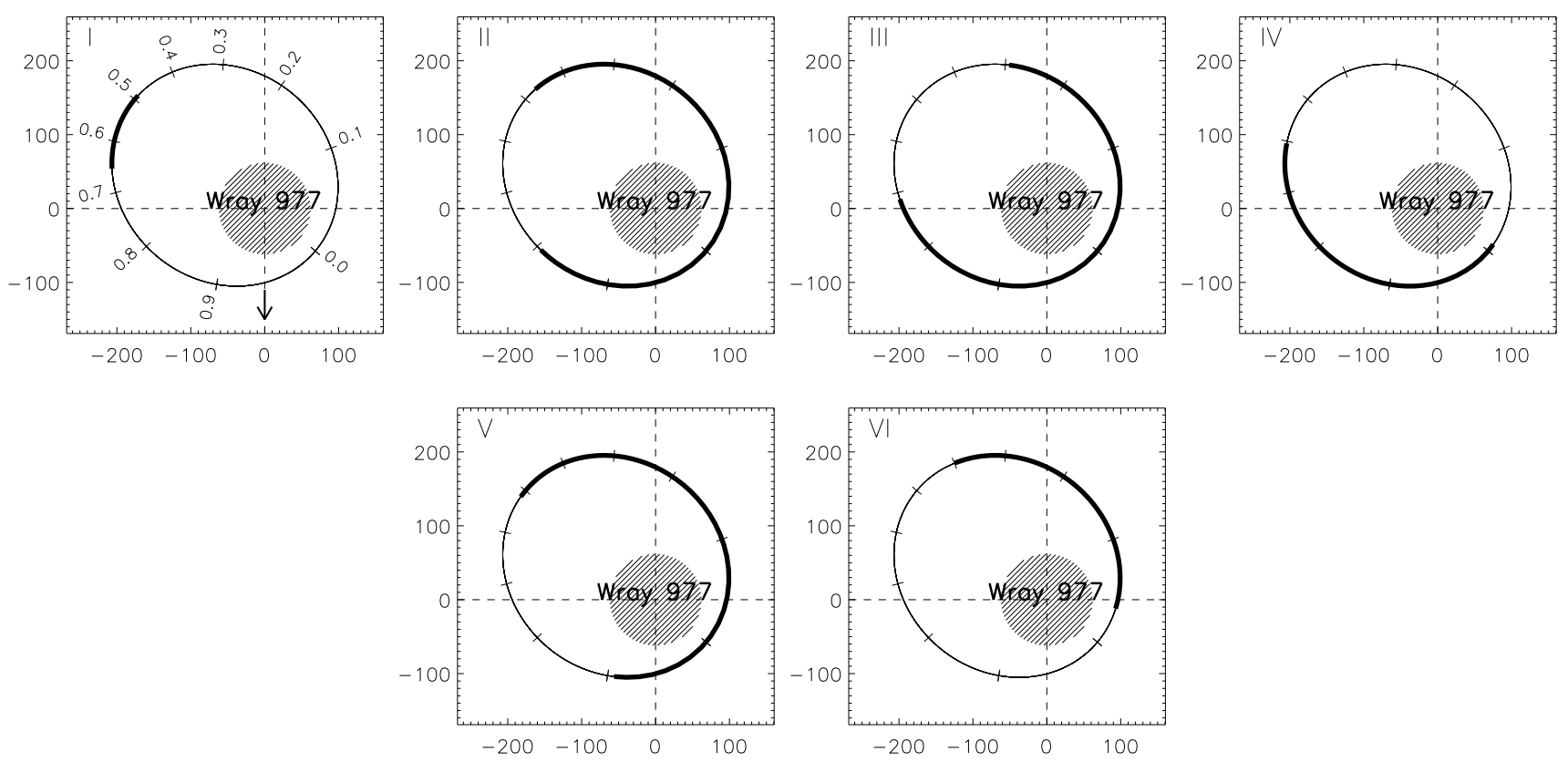

FIG. 1.- Graphical representations of the GX 301-2 binary system. The solid thick line in each panel shows the orbital phases observed by SuperAGILE during the six observation blocks summarized in Table 1 Ticks on the Neutron Star orbits represent the orbital phases with step 0.1 as displayed in the upper-left panel, the NS orbital motion is counter-clockwise. Units along the axis are in solar radii while the arrow in the upper-left panel represents the observer direction. Roman numerals in each panel indicate the Observation ID.

TABLE 1

Journal of the AGILE ObSERVATIONS OF GX 301-2.

\begin{tabular}{cccccc} 
Obs. ID & Mission Phase & MJD Start & MJD Stop & Orbital Phase & Off-axis Angle (deg) \\
\hline I & SVP & 54298.4 & 54305.3 & $0.49-0.66$ & $7-10$ \\
II & SVP, AO1 & 54311.6 & 54339.1 & $0.81-0.47$ & $4-15$ \\
III & AO1 & 54473.6 & 54497.7 & $0.71-0.29$ & $5-18$ \\
IV & AO1 & 54510.6 & 54527.3 & $0.60-0.01$ & $10-17$ \\
V & AO1 & 54647.6 & 54672.9 & $0.91-0.52$ & $14-20$ \\
VI & AO1 & 54694.3 & 54709.5 & $0.03-0.40$ & $12-17$ \\
\hline
\end{tabular}

Note. - First column indicates the Observation ID, the second column specifies the AGILE mission phase: SVP=Science Verification Phase, AO1=Scientific Observations Program pointings (see AGILE Mission Announcement of Opportunity Cycle-1: http://agile.asdc.asi.it/).

(Evangelista et al. 2008) which allows fast and accurate analysis for bright sources. For each AGILE orbit the source count rate was automatically extracted in the energy range 20 $60 \mathrm{keV}$, excluding the time spent by the satellite in crossing the South Atlantic Geomagnetic Anomaly and the time when the Earth occulted the line of sight to the source. Normalized count rates (in units of counts $\mathrm{cm}^{-2} \mathrm{~s}^{-1}$ ) were then obtained by considering the effective area of the exposed portion of the detectors and assuming a Crab-like spectrum (e.g. Frontera et al. 2007). Fluxes in unit of the Crab flux were calculated by applying a normalization factor of 0.15 to the normalized count rates. Uncertainties on the flux measurements were estimated by adding to the statistical error a systematic error equal to the $8 \%$ of the measured flux (Feroci et al. 2009). In order to avoid the introduction of biases in the light curve we did not select the source detections for significance but all the observations were used in the analysis. A detailed description of the method used for the generation of the SuperAGILE light curves can be found in Feroci et al. (2009). The resulting 20$60 \mathrm{keV}$ light curve of GX 301-2 with a $\sim 6 \times 10^{3} \mathrm{~s}$ binning is shown in Figure2,

SuperAGILE events are time-tagged with microsecond precision by the AGILE on-board Pulse-Per-Second (PPS) system, which is synchronized to UTC once a second by the
Global Positioning System (GPS). In order to perform timing analysis all the SuperAGILE events are converted on ground to the Terrestrial Dynamical Time (TDT) reference system. The photon arrival time (TOA) is then converted to Barycenter Dynamical Time and the correction for the arrival delay at the Solar System Barycenter is calculated by using the JPL planetary ephemeris DE405. A more detailed description of the correction algorithm may be found in Pellizzoni et al. (2009). Correction for the time delay introduced by the binary orbital motion was performed using the Blandford-Teukolsky model (Blandford \& Teukolsky 1976; Taylor \& Weisberg 1989). To perform the TOA correction we used the orbital parameters provided by Koh et al. (1997), except for the orbital period $\left(P_{\text {orb }}\right)$ and the time of periastron passage $\left(T_{0}\right)$, for which we utilized the updated values derived by Doroshenko et al. (2008). These parameters are given in Table 2 The quoted uncertainties are given at $1 \sigma$ level throughout the paper, unless otherwise specified.

\section{ANALYSIS OF THE SPIN PERIOD \\ 3.1. Pulse-shape Profile}

A search for the pulsed emission from the NS was performed on the corrected events of all the SuperAGILE source observations using the standard epoch folding technique 


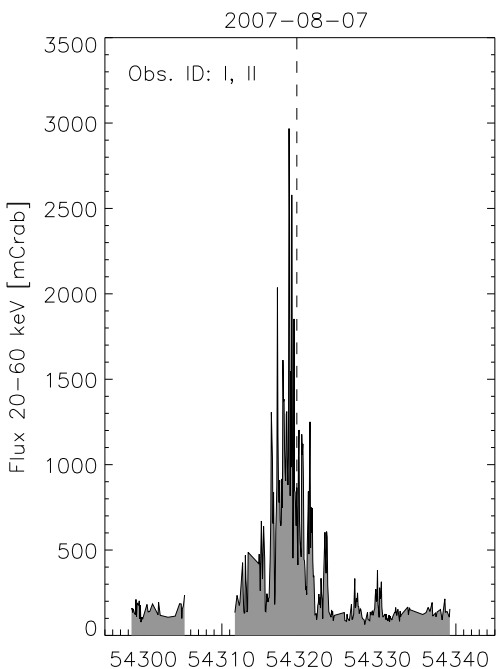

5430054310543205433054340

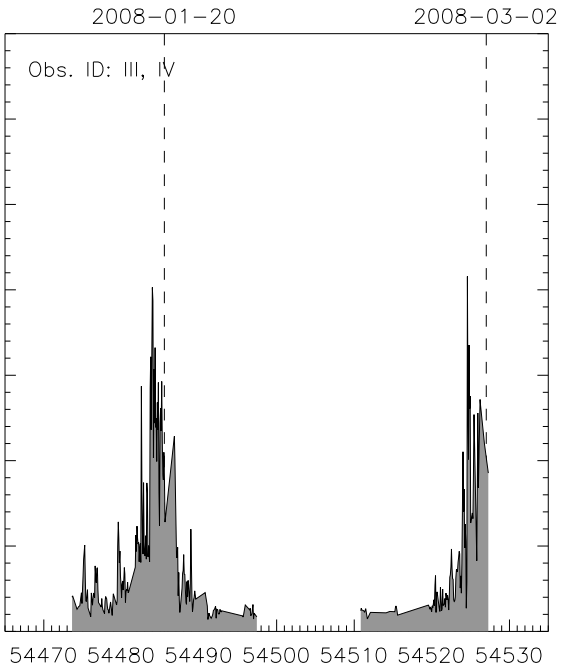

Time [MJD]

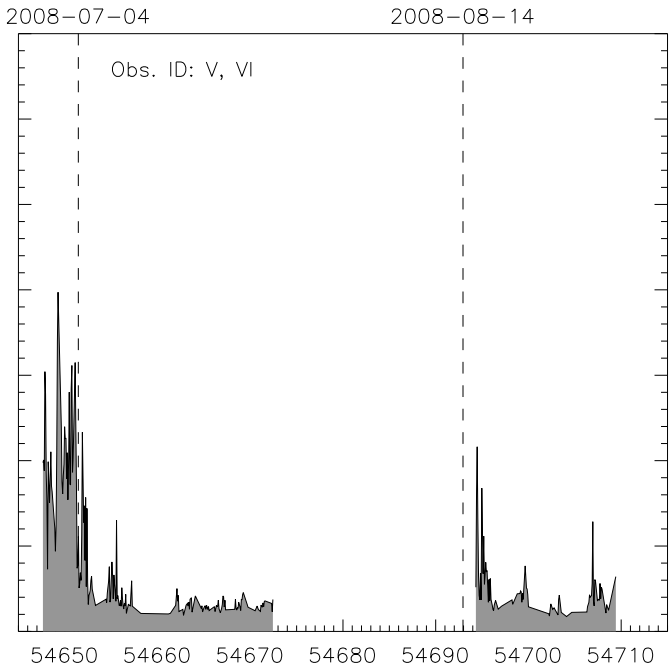

FIG. 2.- Light curve of GX 301-2 as observed by SuperAGILE. The source was monitored for MJD 54298 to MJD 54709. Dashed lines indicate the periastron passages on the basis of the orbital parameters calculated by Doroshenko et al. (2008).

TABLE 2

Orbital Parameters of GX 301-2.

\begin{tabular}{lcc} 
Parameter & Koh et al. (1997) & Doroshenko et al. (2008) \\
\hline$P_{\text {orb }}$ (day) & $41.498 \pm 0.006$ & $41.482 \pm 0.006$ \\
$e$ & $0.462 \pm 0.042$ & $\ldots$ \\
$\mathrm{a}_{x} \sin i$ (lt-s) & $368.3 \pm 11.1$ & $\ldots$ \\
$\omega$ (deg) & $310.4 \pm 4.2$ & $\ldots$ \\
$\mathrm{T}_{0}$ (MJD) & $48802.79 \pm 0.36$ & $53531.63 \pm 0.03$ \\
\hline
\end{tabular}

Note. - Parameters are derived by Kohet al. (1997) and by Doroshenko et al. (2008). The uncertainties are at the $3 \sigma$ level both for the Koh and the Doroshenko parameters.

(Leahy et al. 1983). Due to the different off-axis angles to which the source was observed in the different pointings (see Table 1), corresponding to significantly different exposed effective areas, for each orbital phase bin we selected the available data with the highest signal-to-noise ratio, in order to obtain the best determination of the pulse shape. In case of pulse shape variability between different orbital cycles, this choice biases the pulse shape to the specific selection. However, we verified that in the other SuperAGILE observations of the same orbital phase bin the pulse shape was consistent, within the statistical uncertainties. Following this approach, pulse shape profiles for orbital phases between 0.26 and 0.64 and between 0.81 and 0.06 were obtained from data collected during Observation I and II, while the other pulse shape profiles were built using data taken during Observation III. The width of the phase intervals was chosen according to the signal-to-noise ratio in the profiles. This implies to have a phase bin equal to $\sim 0.10$ ( $\sim 4$ days) for the orbital phase interval 0.06-0.34, where the source flux is lower, while a phase bin size $\lesssim 0.05$ was used for the other orbital phases with higher signal-to-noise. We were not able to detect NS pulsation in orbital intervals $0.16-0.26,0.34-0.38,0.43-0.49$, $0.54-0.59$ and $0.64-0.71$.

Due to the erratic behavior of the spin period of the windfed pulsar even on short timescales, as reported by Nagase (1989), Bildsten et al. (1997), Pravdo \& Ghosh (2001), and taking into account the errors on period determination, we were not able to determine the absolute phasing of the pulse shape profile. Hence, we cross-correlated each pulse profile with a template obtained in the orbital phase 0.96-0.01 (lower-right panel in Figure 3), which represents the pulse shape with the highest signal-to-noise. The resulting phase shifts were then used to build the background subtracted phase-aligned folded light curves shown in Figure 3. Despite the different statistical quality of the curves, a variability along the orbital cycle can be clearly identified. The main peaks change in duration, shape and height. The main peak, at spin phase 0.0 , shows a significant double peak structure only at orbital phases between 0.96 and 0.06 , where the most dramatic flux evolution of the source occurs. Although these are the orbital phases where the statistical quality is higher, the insurgence of the minor peak does not appear as an observational bias: the evolution is gradual and continuous in the three panels between phases 0.91 and 0.06 , all with comparable quality.

Another variability feature that is worth remarking is the behavior of the interpulse regions. At spin phase around $0.7 \mathrm{a}$ small peak appears at orbital phase 0.88-0.91. Its onset seems already anticipated in the previous orbital phase intervals. The other interpulse region, around spin phase 0.3 , shows instead an intensity variability. The source flux in this interval is significantly higher, with respect to the peaks, in the spin phase interval between 0.96 and 0.06 , the same where the small peak mentioned above appears.

\subsection{Secular Evolution of the Spin Period}

The SuperAGILE long-term and nearly continuous monitoring of GX 301-2 allowed us to measure the spin period at different epochs and put it in the context of the secular timing evolution of the source, as available from the literature. Figure 4 (a) shows the spin period $P$ as a function of the time from the discovery of the pulsed emission by White et al. (1976) to the last SuperAGILE observation (MJD 54709.5). This plot confirms that GX 301-2 is subject to unpredictable torque variations superimposed on long-term smooth variations of the spin period. During the last $\sim 30$ years the spin period changed passing from a spin-up state, during which $P$ decreased from $\sim 700.5 \mathrm{~s}$ to $\sim 675.4 \mathrm{~s}$, to a spin-down condition reaching the current value of $P \sim 687.3$ s. The Figure clearly shows the two rapid spin-up episodes identified by Koh et al. (1997) analyzing CGRO/BATSE observations between MJD 48440 and 

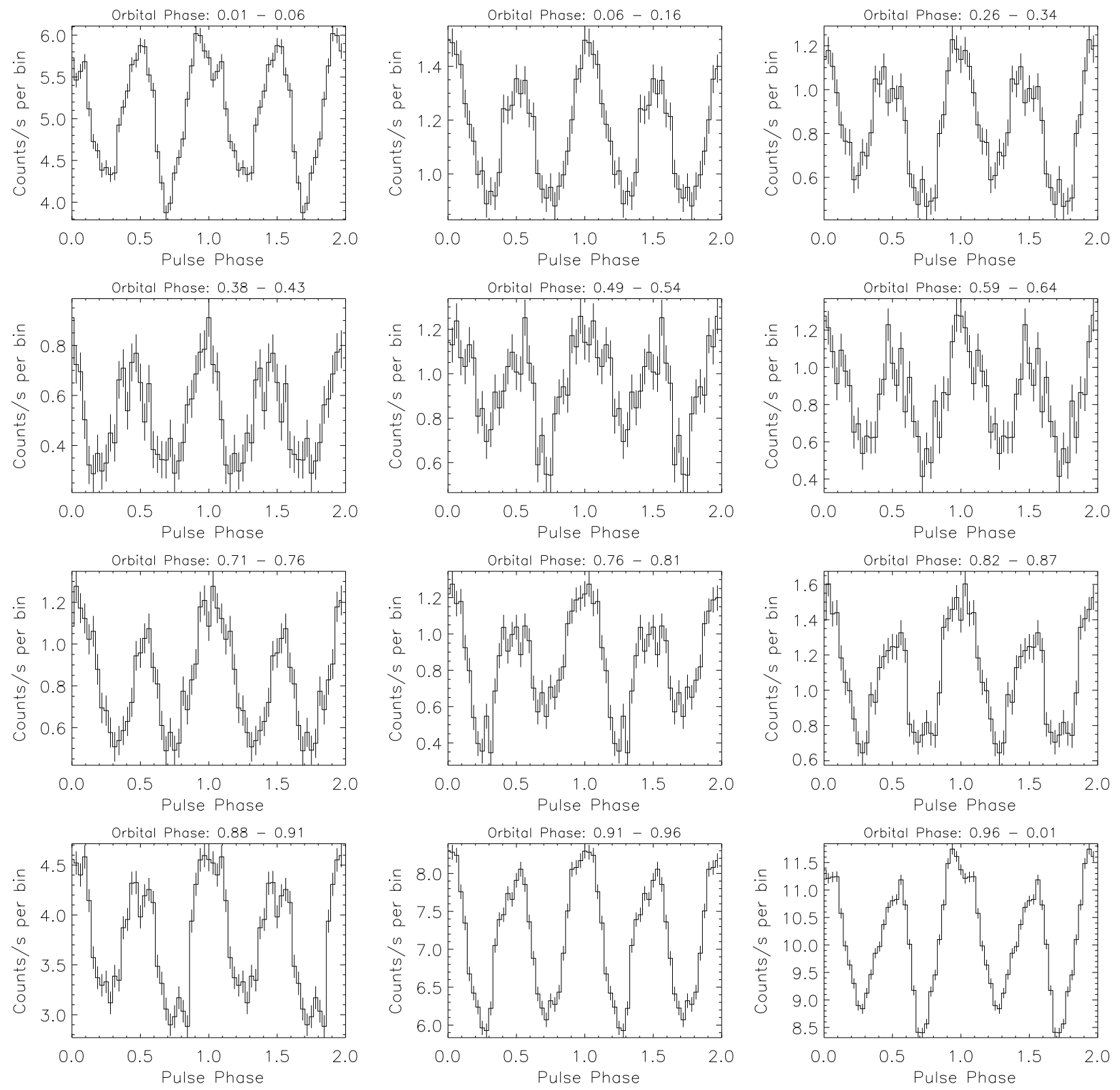

FIG. 3.- GX 301-2 pulse shape profiles. Each panel represents the pulse shape profile, after the background subtraction, in an orbital phase interval as reported on top of each figure. See the text for a detailed description of the methods used to obtain the pulse shape profiles.

MJD 48463 and between MJD 49230 and MJD 49245. Comparing BATSE $23, R X T E / P C A$ (Kreykenbohm et al. 2004), INTEGRAL (Doroshenko et al. 2008) and SuperAGILE data in Figure 4 (a) we find evidence of at least two other spin-up episodes, occurred between the observations at MJD 51830 and MJD 52789, and around MJD 54000.

Figure 4 b) displays the secular variation of the GX 301-2 pulse period during the SuperAGILE observations. $P$ varies in the range $685.3-688.0 \mathrm{~s}$ in about 410 days. We find a period evolution consistent with the constant spin down rate of $4.0 \pm 0.3 \times 10^{-8} \mathrm{~s} \mathrm{~s}^{-1}$. However, the short term variability of the period clearly shows significant timing noise around the general trend, and the linear fit (dashed line in Figure 4(b)) is indeed a poor modeling of the period evolution, leading to a reduced $\chi^{2}$ value of 2.9 (34 dof).

\footnotetext{
${ }^{23}$ http://gammaray.msfc.nasa.gov/batse/pulsar
}

\section{ORBITAL VARIABILITY}

\subsection{SuperAGILE and RXTE/ASM Folded Light Curves}

Using all the SuperAGILE data collected during the observations listed in Table 1, we obtained the complete, folded orbital light curve of GX 301-2 shown in the top panel of Figure 5. The orbital parameters $\left(P_{\text {orb }}, T_{0}\right)$ used in the folding procedure were the same used for the correction for the time delay discussed in Section 3. The difference in the orbital solution found by Doroshenko et al. (2008) and by Koh et al. (1997) results in a 5\% phase shift of the PP flare in the orbital folding, while the shape of the folded light curve does not change noticeably. In order to obtain a folded light curve in the soft X-ray band we used public data from the All Sky Monitor onboard RXTE (Levine et al. 1996). ASM data for GX 301-2 cover the time span from MJD 50087.285 to MJD 54720.745 and consist of source count rates for each 


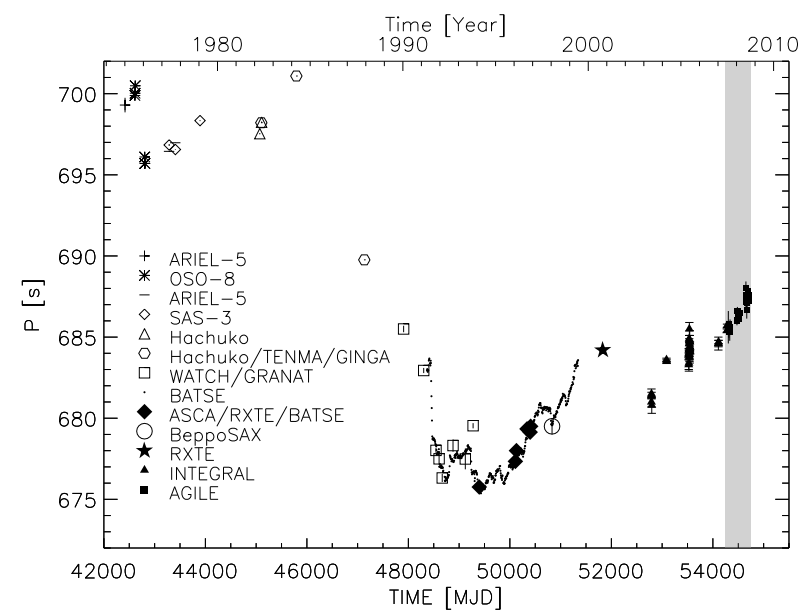

(a)

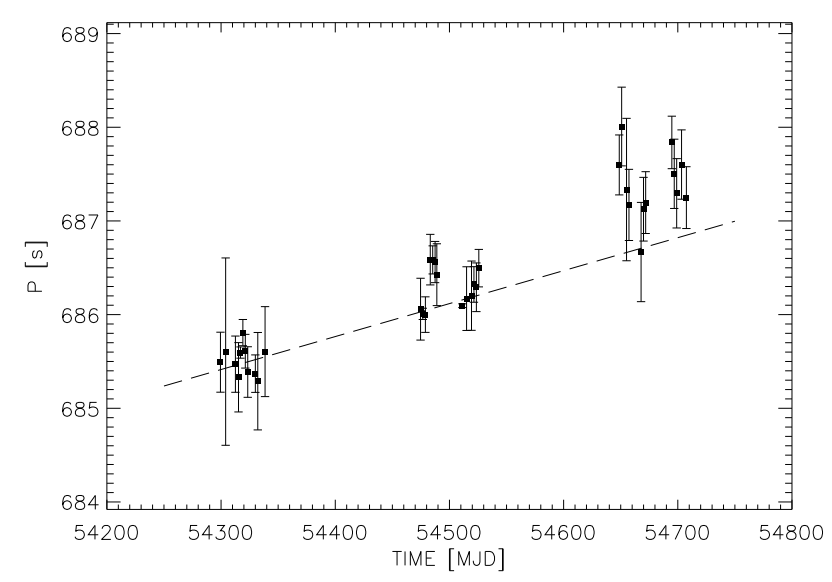

(b)

FIG. 4.- (a) Secular variations of GX 301-2 pulse period. Data are from the following references: cross symbols (White et al. 1976), asterisks (Swank et al. 1976), dashes (White et al. 1978), diamonds (Kelley et al. 1980), triangles (Kawai et al. 1985), hexagons (Nagase 1989), squares (Chichkov et al. 1995), filled diamonds (Pravdo \& Ghosh 2001), stars (Krevkenbohm et al. 2004), circles (La Barbera et al. 2005), points (http://gammaray.msfc.nasa.gov/batse/pulsar), filled triangles (Doroshenko et al. 2008), filled squares in highlighted region (SuperAGILE data). (b) Zoomed view of the SuperAGILE data. The dashed line represents the best-fit secular variation of the pulse period assuming a linear trend.

ASM dwell ( 1 dwell $=90 \mathrm{~s}$ observation $)$ in the band $2 \mathrm{keV}$ $12 \mathrm{keV}$. The dwell-by-dwell data were filtered according to the criteria reported on $R X T E / A S M$ web site 24 and then a light curve was built from the cleaned data. The ASM folded light curve for GX 301-2 is displayed in the second panel of Figure 5 (solid line).

Both the SuperAGILE and the ASM light curves are given in units of mCrab. We used the standard value of $1 \mathrm{Crab}=75$ counts $\mathrm{s}^{-1}$ as a conversion factor for the ASM count rates, while the SuperAGILE normalization is 0.15 counts $\mathrm{cm}^{-2} \mathrm{~s}^{-1}$. The two folded orbital light curves clearly show the PP flaring activity, with an intensity peak observed at orbital phase between $\sim 0.9$ and $\sim 0.0$ and a full width at half maximum of about 0.1 in phase. Fitting the PP flare by using a Gaussian function plus a constant term, we obtained a value of orbital phase of $0.954 \pm 0.005$ for the flare position, with a FWHM value of 0.109 . The remaining or-

\footnotetext{
${ }^{24}$ http://heasarc.gsfc.nasa.gov/docs/xte/SOF/asmlc.html
}

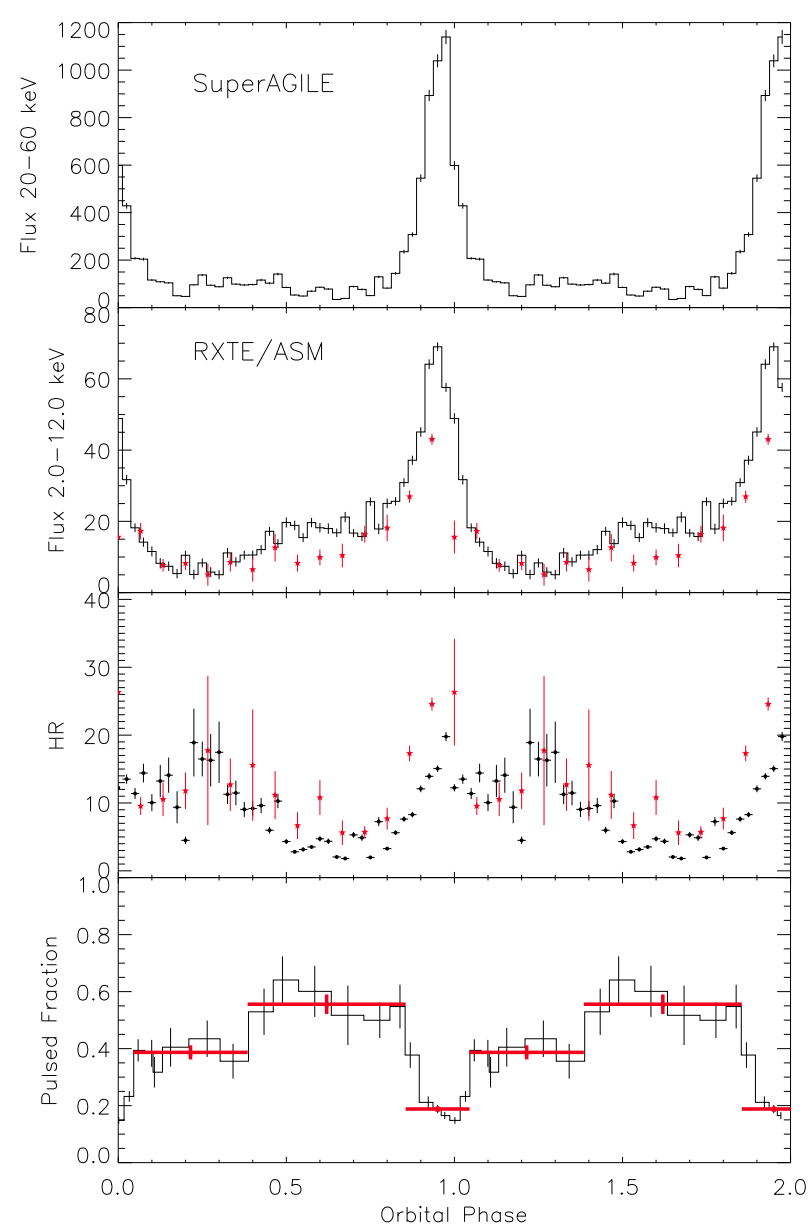

FIG. 5. - Top panel: SuperAGILE orbital light curve $(20-60 \mathrm{keV})$ of GX 301-2 folded at orbital period $P_{\text {orb }}=41.482$ d. Second panel: $R X T E / A S M$ orbital folded light curve (2-12 keV) of GX 301-2 obtained using the whole ASM dataset from MJD 50087.285 to MJD 54720.745 (black solid line) and only the observations contemporaneous to SuperAGILE (red stars). Both the folded light curves are given in mCrab units. Third panel: Hardness ratio (20-60 keV to $2-12 \mathrm{keV}$ ) calculated using the whole SuperAGILE and ASM dataset (filled circle) and the contemporaneous observations only (red stars). Bottom panel: pulsed fraction $\left(P_{\text {frac }}\right)$ as a function of the orbital phase. Thick points represent the mean pulsed fraction values in the orbital phases 0.05 $0.39,0.39-0.86$ and $0.86-0.05$. The sharp $P_{\text {frac }}$ minimum which corresponds with the pre-periastron flare is clearly visible.

bital interval exhibits a different behavior in the hard and soft X-ray data: in the $20-60 \mathrm{keV}$ energy band a nearly constant persistent emission is detected between phases 0.1 and 0.8 , while in the same phase interval the soft X-ray data show a broad hump, covering phases between 0.5 and 1.0.

\subsection{Variability of the pulsed fraction}

The variability in the shape of the pulse as a function of the orbital phase shown in Figure 3 motivated us to study the pulsed fraction across the binary orbital cycle, to determine its possible variability and correlation with other source parameters, such as flux and hardness. We operationally define the pulsed fraction $\left(P_{\text {frac }}\right)$ as the ratio between the pulsed counts, as derived directly from folding the event list, including photons from the background and from other sources in the FoV, and the total counts determined by the imaging procedure, including the counts from GX 301-2 only. We performed the analysis of the pulsed fraction as a function of the pulsar or- 
TABLE 3

Mean Hardness Ratio of the PP Flares

\begin{tabular}{ccccc} 
MJD Start & MJD Stop & Phase Start & Phase Stop & HR \\
\hline 54315.84 & 54319.37 & 0.91 & 0.99 & $10.4 \pm 0.6$ \\
54481.77 & 54485.30 & 0.91 & 0.99 & $17.5 \pm 1.2$ \\
54523.25 & 54526.78 & 0.91 & 0.99 & $21.8 \pm 1.7$ \\
54647.70 & 54652.22 & 0.91 & 0.99 & $14.0 \pm 0.9$ \\
\hline
\end{tabular}

bital phase for all the available SuperAGILE observations during which the pulsation was detected. The results are shown in the bottom panel of Figure 5] where $P_{\text {frac }}$ is plotted as a function of the orbital phase.

$P_{\text {frac }}$ exhibits large variations across the binary cycle, passing from a minimum of $P_{\text {frac }}=0.15 \pm 0.02$ at orbital phase $\sim 0.0$ to a maximum of $P_{\text {frac }}=0.64 \pm 0.12$ at orbital phase $\sim 0.5$. With the aim of quantifying the significance of the variability of the pulsed fraction, we took the average of $P_{\text {frac }}$ in the phase intervals where it appears consistent with a constant value, that is $0.05-0.39,0.39-0.86$ and $0.86-0.05$. Thick red points in the bottom panel of Figure 5 represent the mean $P_{\text {frac }}$ values calculated during the three selected orbital intervals. The values in the three phase intervals are $0.188 \pm 0.009$, $0.387 \pm 0.025$ and $0.556 \pm 0.034$, ordered by phase. This analysis shows that it is possible to identify three different "states" in the pulsed fraction as a function of the NS orbital motion, with the intermediate one occurring just after the PP flares and lasting approximately up to the phase corresponding to the superior conjunction of the binary system.

The bottom panel in Figure 5 shows that the pulsed fraction reaches its minimum at the phase when the large flare occurs, that is where the largest variations in the pulse shape are detected. We then investigated the possible dependence of $P_{\text {frac }}$ on the source flux. The results are shown in Figure 6a), where the value of the pulsed fraction against the source flux is displayed, independently of the orbital or spin phase of the source. A clear anti-correlation between these two quantities is found, confirming it as a general feature and not only an event occurring during the PP periodic flare. The plot shows that the pulsed fraction decreases rapidly at increasing fluxes, reaching a plateau for large fluxes corresponding to a pulsed fraction of $15 \%$, approximately a factor 4 smaller than the maximum value. A similar behavior was also recently reported by Lutovinov \& Tsygankov (2009) using INTEGRAL/IBIS data.

\subsection{Hardness Ratio Versus Orbital Phase}

Given the different orbital flux modulation at soft and hard $\mathrm{X}$-rays (Figure 5), we investigated the period-averaged time behavior of the source hardness along the orbital light curve. To this aim we computed an hardness ratio (HR) by using the flux measurements by SuperAGILE and ASM, that is between the energy ranges $20-60 \mathrm{keV}$ and $2-12 \mathrm{keV}$. The third panel in Figure 5 shows the result. Filled circles indicate the orbital phase-resolved hardness ratio obtained by using the complete temporal data set from both experiments. Moreover, star-like symbols provide the same quantity, but computed after selecting the ASM time intervals coeval with the SuperAGILE data sets (the ASM light curve with this selection is shown using the same symbols in the second panel of the same figure).

The time profile of the HR computed using both time selections indicates a clear modulation of the source spectral hardness as a function of the orbital phase, where we identify the following features. A spectral hardening is detected

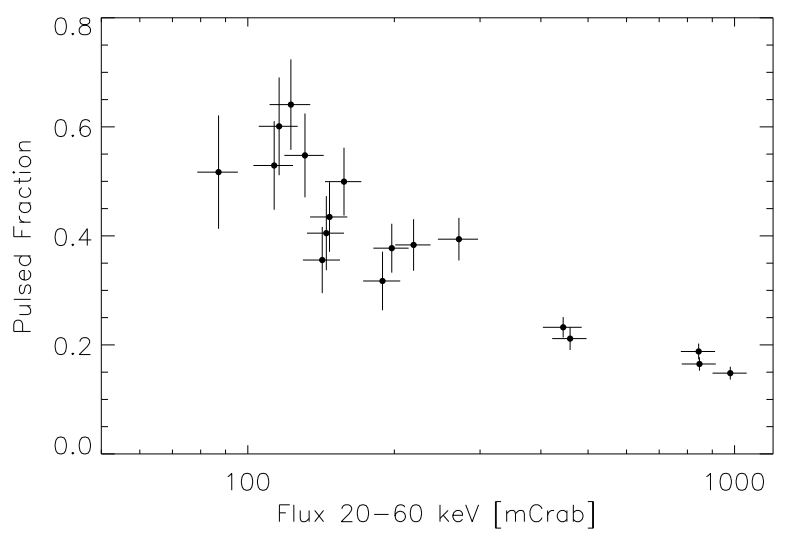

(a)

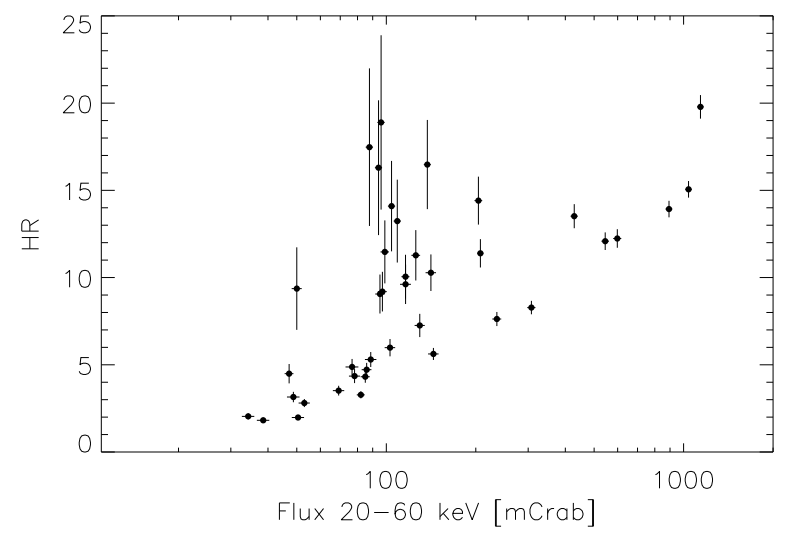

(b)

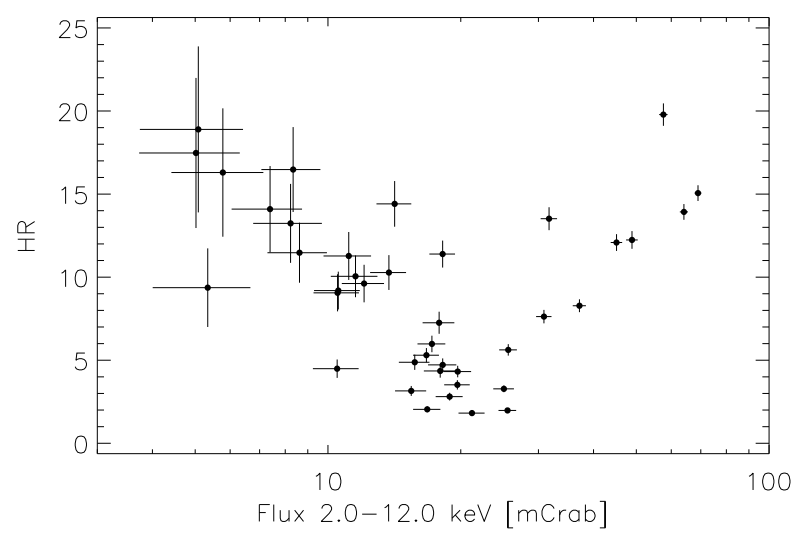

(c)

FIG. 6.- (a) $P_{\text {frac }}$ as a function of the source intensity. The clear anticorrelation between pulsed fraction and source flux is ascribed to the different amount of matter which accumulates near and accretes onto the neutron star. (b) and (c) Hardness ratio as a function of the source flux in the $20-60 \mathrm{keV}$ (b) and 2-12 keV (c) energy bands.

during the pre-periastron flare: the hard X-ray flux rises faster than the soft X-rays, and lasts longer. Then, when the soft $\mathrm{X}$-ray flux drops at its minimum orbital level, around phase 0.2 , the hard X-rays hold their intensity level, indicating an increasing hardness of the emitted radiation. Subsequently, the rise of the soft X-ray flux after phase 0.5 (the apastron) is not accompanied by a corresponding increase at hard Xrays, indicating a softer emission in this part of the orbit. We 
note that using the simultaneous data only (stars in the HR plot), the above features are confirmed and, within the limits of the lower statistical quality, emphasized. Actually, the source spectral hardness changes also from one binary orbit to another. In Table 3 we provide the HR values computed in the orbital phases including the PP flare in the four available SuperAGILE observations. The value of the HR changes up to a factor of 2 .

\section{DISCUSSION}

The pointing strategy of the AGILE mission offered SuperAGILE the opportunity of studying the temporal properties of GX 301-2 in the hard X-rays over repeated monthlong stretches of time, covering six 41.5 days binary orbits at different phases. We observed a modulation of the hard $\mathrm{X}$-ray flux along the orbit in general agreement with what reported over the past two decades by previous experiments in soft and hard X-rays. Fitting the source spectrum in the 20-60 keV energy band with a cut-off power-law with photon index fixed to $\Gamma=1.05$ (La Barbera et al. 2005), we obtain $E_{\text {cut }}=24.4_{-1.4}^{+1.2} \mathrm{keV}$ and $E_{\text {fold }}=7.8_{-0.7}^{+0.7} \mathrm{keV}$ for the PP flare occurred around MJD 54316. Using these parameters, and assuming a source distance of $3 \mathrm{kpc}$ (Kaper et al. 2006), we convert the SuperAGILE counts to physical units obtaining a luminosity of $\sim 2.3 \times 10^{37} \mathrm{erg} \mathrm{s}^{-1}$. Under the same assumptions, the luminosity during the quiescent orbital phases is approximately one order of magnitude smaller.

The only complete folded orbital light curve of GX 3012 at energies above $20 \mathrm{keV}$ available in the literature, to which we can compare the SuperAGILE results, was built from the data of the BATSE experiment, using the Earth occultation technique (Pravdo et al. 1995; Pravdo \& Ghosh 2001) or the pulsed flux measurements (Koh et al. 1997). The SuperAGILE data show significant differences. In contrast to the BATSE observations in the same energy range (Pravdo \& Ghosh 2001), the SuperAGILE data do not show any secondary peak in the folded light curve near the passage at the apastron (orbital phase $\sim 0.5$ ). This second peak was indeed weakly detected in the BATSE measurements of the total source flux (Pravdo \& Ghosh 2001), while it was outstanding in the pulsed flux only (Koh et al. 1997). The different shape of the orbital light curve of the total flux as measured by BATSE and SuperAGILE may be ascribed to intrinsic time variability between the different orbits and/or epochs of the observations (1991-1997 for BATSE and 2007-2008 for SuperAGILE) and/or to the different effective bandpasses of the two experiments (harder for BATSE than for SuperAGILE despite their similar nominal energy range). Actually, hints toward time variability near the apastron phase were already provided by the epoch-resolved BATSE folded light curves reported by Pravdo \& Ghosh (2001), where only two out of four curves show a broad hump around phase 0.5 , although the statistical quality of the data does not allow for a definite statement. Also the recent Swift/BAT data of three orbital cycles reported by Pestalozzi et al. (2009) show bumps around phase $\sim 0.5$ in two contiguous orbital cycles around 2008 December (unfortunately these time periods were not covered by SA observations).

Instead, the pronounced bump around the apastron phase found by Koh et al. (1997) in the pulsed flux appears consistent with our results, when the behavior of the pulsed fraction along the binary orbit is considered. As we show in Figure 5. we found a large orbital variation of the pulsed fraction: within the statistical accuracy of our data, the pulsed fraction exhibits a step-like behavior along the orbit. The minimum value is reached at the periastron, while a broad maximum, nearly 3 times as high, is reached at phases between 0.4 and 0.9. This implies that the pulsed flux increases in the phase range corresponding to the apastron, coherently with what was found in the BATSE pulsed data.

Interestingly, the same orbital phases where the pulsed fraction is highest are also those where the emitted radiation is softest. In fact, we analyzed the behavior of the source hardness along the orbit by using the SuperAGILE data in combination with the public $R X T E / A S M$ data. We found that the radiation emitted by the source is harder near the periastron and at phases around $0.2-0.3$, while it becomes significantly softer at phases $0.5-0.8$ (Figure 5). The hardening around phase $0.2-0.3$ was also detected by Pravdo \& Ghosh (2001) through an analysis similar to ours, using the BATSE and ASM data, but they did not report evidence for the hardening near the periastron, that is instead the main feature in our data. More in general, our data suggest an anti-correlation between the hardness and the pulsed fraction at hard X-rays (as well as between the pulsed fraction and the flux, Figure 6 a)).

The general behavior of the source is summarized in the synoptic panels of Figure 7, where the parameters are graphically displayed along the binary orbit. These plots show that even if the only significant feature in the flux modulation observed by SuperAGILE occurs at the pre-periastron phase, the emission mechanism undergoes important changes also in other parts of the orbit. The passage at the periastron is accompanied with a hardening of the radiation and with a sudden and large decrease of the pulsed fraction, as already reported by La Barbera et al. (2005), using the BeppoSAX observations, and Lutovinov \& Tsygankov (2009) using INTEGRAL data. The pulsed fraction shows (Figure 6) a)) a clear anti-correlation with the source flux, thus presumably with the accretion rate. Instead, the hardness ratio does not show a clear dependence on the X-ray flux (and then the accretion rate), suggesting a more complex relation. It is interesting to note that the largest drop in the pulsed fraction is approximately a factor of 3 , from $\sim 0.55$ to $\sim 0.2$, while the flux increase in the same phase interval is about a factor of 10 . This implies that the excess hard radiation emitted during the preperiastron flare does not represent a proportional luminosity increase of the same regions emitting in the preceding orbital phases, that would leave the pulsed fraction unchanged, nor an unrelated emission from a site not subject to occultation by the NS upon spinning, that would cause a larger drop in the pulsed fraction. Thus, either the site of the excess emission is only partially occulted to the line of sight as the NS rotates, or it is associated with an intervening radiative transfer mechanism able to smear the coherence of the photon arrival times (as proposed by Makino et al. 1985). Moving from the periastron to the apastron, the pulsed fraction seems to recover gradually to its maximum value. Instead, the hardness ratio shows a further, smaller peak at phases near 0.25 mostly due to a depression in the soft X-ray flux. Thus the cause intervening in obscuring or depressing the emission of soft X-rays where the NS approaches the apastron phase, leaves the persistent hard X-ray component unaffected, as witnessed by the unchanging flux level measured by SuperAGILE.

Leahy (2002) (see also Leahy \& Kostka 2008) quite convincingly modeled the orbital modulation of the soft X-ray emission in the GX 301-2 system as driven by wind accretion plus a mass stream, drawing an Archimedes spiral path 


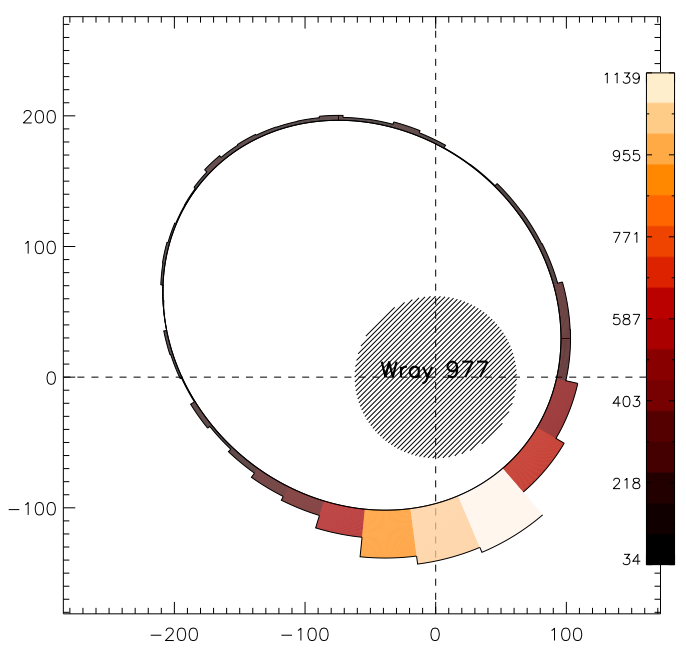

(a)

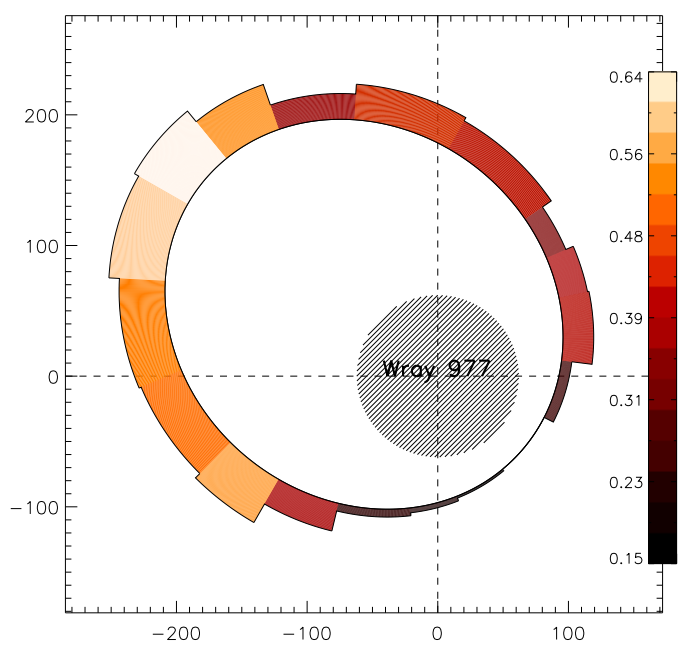

(b)

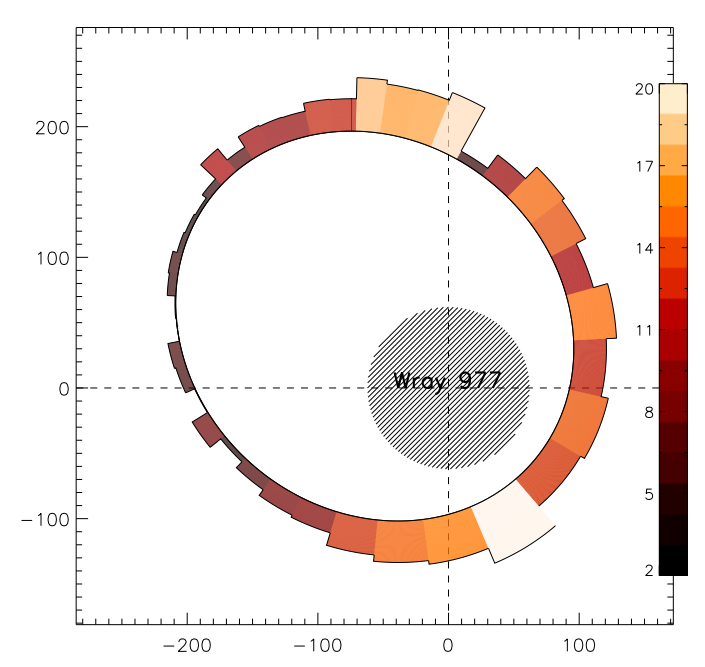

(c)

FIG. 7.- Summary of the orbital behavior of GX 301-2: hard X-ray flux (a), pulsed fraction (b) and hardness ratio (c). Units along the axis are in solar radii.

and periodically intercepting the neutron star. The effect of the spiral stream accretion on the orbital light curve is the pre-periastron flare and the broad bump around the apastron phase, both caused by the sudden and large increase in the accretion rate. The larger density and narrower geometrical size of the intercepted accretion stream near the periastron phase causes a flare shorter and more intense than at the apastron phase. In this scenario, the same matter that accretes is also responsible for a very large absorption column (thus the hardening) and for scattering of the radiation from the neutron star, bringing loss of timing coherence (decrease in the pulsed fraction). This is indeed what the ASM data show at soft X-rays. In the same model, the wind component determines a smooth variation of the flux, peaking only at the pre-periastron phase. This is what the SuperAGILE data show at hard X-rays.

Leahy \& Kostka (2008) show that around phase 0.25 the soft X-rays are largely absorbed by a partial occultation of the neutron star by the atmosphere of the companion star. This is consistent with both the ASM flux modulation and with the SuperAGILE/ASM hardness ratio. However, we observe an even more intense hardening peak of the emitted radiation at the pre-periastron that is not shown in the model prediction by Leahy \& Kostka (2008). In this case the hardness peak is not due to a decrease in the soft X-ray flux, but to a larger increase in the hard X-rays than in soft X-rays, still in the presence of a strong flare in both energy bands. If absorption is responsible for such an effect, this implies that the site of the large flare lies behind the absorbing material (thus the unabsorbed soft X-ray flux would be far larger) and both the flare and absorption occur at the same orbital phase, suggesting that the two are related in origin. Large increases (factor of $\sim 10$ ) in the absorption column near the periastron phase were indeed reported by early studies (Haberl 1991 using EXOSAT data, Leahy 1991 using TENMA data) while more recent studies, using a partial covering absorber model, reported smaller variations (factor of 2 with BeppoSAX, La Barbera et al. 2005) and rather scattered in orbital phase (Mukherjee \& Paul 2004, with $R X T E / \mathrm{PCA})$.

As already mentioned, at this phase we detect a large decrease in the pulsed fraction, implying that the hard X-rays 
here are more loosely affected by the NS spinning than the soft $\mathrm{X}$-rays. This may suggest that (at least) the hard X-ray emission at the pre-periastron peak may be associated with temporary conditions setting up when the NS crosses the accretion stream at its minimum distance from the companion star. It is also interesting to remind that just at this orbital phase, a detection of non-thermal radio emission from GX 301-2 has been very recently reported by Pestalozzi et al. (2009).

Changes of the properties or sites of the hard X-ray emission along the orbit are suggested also by our study of the neutron star pulse profile as a function of the orbital phase. Similar to what found by La Barbera et al. (2005) using the BeppoSAX data, we detected a large variability in the pulse shape of the pulsar in the hard X-ray range. The main points of variability we detected are: the structure of the main peak, the intensity at the interpulse and the amplitude of the pulsation (i.e., the pulsed fraction discussed above). As found by BeppoSAX, in the SuperAGILE data the pulse appears smoothest at orbital phases near the pre-periastron. The shape we obtained for the orbital phase interval 0.91-0.96 is reminiscent of the BeppoSAX/PDS curve. However, in approaching and leaving the phase of the large flare, the pulse shape shows transient features. A small peak structure (at spin phase around 0.1 in Figure 3 gradually appears on the rising and decaying phases of the flare (orbital phases from 0.91 to 0.06), being more prominent after the periastron. Simultaneously, the flux level at the inter-pulse phase (around 0.25) becomes higher. As the neutron star approaches the apastron and goes beyond, the pulse shape at energies above $20 \mathrm{keV}$ becomes increasingly complex (although the smaller statistics does not allow us to identify individual substructures), with the main pulses becoming alternately narrower and wider. Interestingly, approaching again the pre-periastron phase, a small peak appears in the interpulse region. This clearly shows up at orbital phase $0.88-0.91$. All of these features, including the smaller peak, are not in contrast with the BeppoSAX results, obtained by La Barbera et al. (2005) in their detailed analysis of the pulse shape in four short orbital phase intervals. Altogether these results seem to suggest that the emitting region on the neutron star is significantly affected by the changing accretion pattern along the binary orbit.

One last consideration derives from the secular variation of the pulse period of the neutron star, collected in Figure 4 As already commented and discussed by several authors (e.g. Koh et al. 1997), the spin history is complex and shows several changes in the spin-up and spin-down trends, sometimes also very rapid. The major changes in the past were probably accompanied to important variations in the emission. In the last $\sim 15$ years (from MJD 49500 onward), a general spindown trend is settled, perturbed only by few short and rapid spin-up episodes. In particular, despite some small-amplitude timing noise that SuperAGILE detected (Figure 4(b)), in the last $\sim$ two years the GX 301-2 system appears rather steadily evolving with a spin-down rate of about $1.3 \mathrm{~s} \mathrm{yr}^{-1}$, showing no rapid or large perturbations.

\section{SUMMARY AND CONCLUSIONS}

We studied the long term behavior of the HMXB GX 301-2 using the hard X-ray data collected by SuperAGILE during the first year of the AGILE mission. The observations, carried out in the $20-60 \mathrm{keV}$ energy band, have a large net exposure, about $3.7 \mathrm{Ms}$, and cover all the orbital phases of the binary system. The SuperAGILE data offer one of the most complete hard X-ray monitoring of the 41.5-day long binary period available to date. The data span covers large fractions of six orbital cycles. The secular trend of the $\sim 680 \mathrm{~s}$ pulse period is consistent with the previous observations, although the SuperAGILE data show a decrease in the spin-down rate with respect to the observations reported by Doroshenko et al. (2008). The spin-down trend is approximately constant, but there is indication of a significant timing noise, of the order of $\sim 0.2 \%$.

Complementing the SuperAGILE data with those of RossiXTE/ASM, the source behavior was characterized at all the phases of the binary orbit, in terms of its soft and hard Xray flux, spectral hardness, spin period history, pulsed fraction and pulse shape. Compared with the soft X-ray data, the hard $\mathrm{X}$-ray lightcurve exhibits a smaller modulation of the emission during the orbital phases $0.1-0.8$ and a larger intensity of the pre-periastron flare. This results in a spectral hardness which presents clear orbital modulation, with peaks in correspondence with the pre-periastron flare and near phase 0.25. The timing analysis of the hard X-ray emission showed a variable pulse shape profile as a function of the orbital phase, with substructures detected near the passage at the periastron, and a clear modulation of the pulsed fraction, which appears in turn strongly anti-correlated with the source intensity.

Overall, the scenario depicted by the SA data appears generally consistent with the model for this binary system proposed by Leahy \& Kostka (2008). The predictions of the model are based on the variability in the accretion rate caused by the orbital motion of the NS in the environment of the matter outflow from the companion star. The model is suited to the low energy observations by $R X T E / \mathrm{ASM}$, for which it reproduces the peaked flare at the pre-periastron and the broader peak at the apastron. They both are related to the NS crossing of the accretion stream, the difference between the two being mostly in the local density and geometrical size of the stream at the crossing point. The higher accretion rate causes a soft $\mathrm{X}$-ray brightening, and scattering of the radiation by the same matter would cause loss of coherence and then a decrease in the pulsed fraction. In the hard X-rays we do not detect the broad peak at the apastron phase (although other hard X-ray observations with BATSE and Swift/BAT suggest time variability for it). Also, our observation of the folded hardness ratio (20-50 keV to $2-12 \mathrm{keV}$ ) along the orbit is not fully consistent with the model expectations. Indeed the model predicts an increase in the column density near the apastron phase, but it does not predict a similar increase near the pre-periastron, that we instead clearly detect.

More in general, our results fit in the overall scenario of the binary X-ray pulsars (e.g. Parmar et al. 1989), where the timing properties are related to the source luminosity. As a matter of fact, our analysis shows that the pulsed fraction is clearly anti-correlated with the source intensity, allowing us to attribute the accretion rate the role of driving parameter in the system. However, while the timing properties appear rather well correlated with the source intensity, the same is not true for the hardness ratio, for which we cannot identify a trend with the flux. This implies that the emitting process and/or geometry change along the orbit in a way that is not immediately associated to the variation of the accretion rate only.

We thank L. Stella, P. Casella and R. Campana for useful discussions and for support in data interpretation. The AGILE Mission is funded by the Italian Space Agency (ASI) with sci- 
entific and programmatic participation by the Italian Institute of Astrophysics (INAF) and the Italian Institute of Nuclear Physics (INFN). We acknowledge the use of public data from the RXTE/ASM data archive.

\section{REFERENCES}

Bildsten, L., et al. 1997, ApJS, 113, 367

Blandford, R., \& Teukolsky, S. A. 1976, ApJ, 205, 580

Chichkov, M. A., Syunyaev, R. A., Lapshov, I. Y., Lund, N., Brandt, S., \& Castro-Tirado, A. 1995, Astronomy Letters, 21, 435

Doroshenko, V., Staubert, R., Kreykenbom, I., Santangelo, A., \& Ferrigno, C. 2008, in Proceedings of the 7th INTEGRAL Workshop

Evangelista, Y., et al. 2008, in Society of Photo-Optical Instrumentation Engineers (SPIE) Conference Series, Vol. 7011, Society of Photo-Optical Instrumentation Engineers (SPIE) Conference Series, 70113B-1

Feroci, M., et al. 2007, Nuclear Instruments and Methods in Physics Research A, 581, 728

-. 2009, A\&A, in press

Frontera, F., et al. 2007, ApJ, 666, 86

Haberl, F. 1991, ApJ, 376, 245

Kaper, L., van der Meer, A., \& Najarro, F. 2006, A\&A, 457, 595

Kawai, N., Makishima, K., Matsuoka, M., Mitani, K., Murakami, T., \& Nagase, F. 1985, PASJ, 37, 647

Kelley, R., Rappaport, S., \& Petre, R. 1980, ApJ, 238, 699

Koh, D. T., et al. 1997, ApJ, 479, 933

Kreykenbohm, I., Wilms, J., Coburn, W., Kuster, M., Rothschild, R. E., Heindl, W. A., Kretschmar, P., \& Staubert, R. 2004, A\&A, 427, 975

La Barbera, A., Segreto, A., Santangelo, A., Kreykenbohm, I., \& Orlandini, M. 2005, A\&A, 438, 617

Lazzarotto, F., et al. 2008, in Astronomical Society of the Pacific Conference Series, Vol. 394, Astronomical Data Analysis Software and Systems XVII, ed. R. W. Argyle, P. S. Bunclark, \& J. R. Lewis, 593

Leahy, D. A. 1991, MNRAS, 250, 310

-. 2002, A\&A, 391, 219

Leahy, D. A., Elsner, R. F., \& Weisskopf, M. C. 1983, ApJ, 272, 256
Leahy, D. A., \& Kostka, M. 2008, MNRAS, 384, 747

Levine, A. M., Bradt, H., Cui, W., Jernigan, J. G., Morgan, E. H., Remillard, R., Shirey, R. E., \& Smith, D. A. 1996, ApJ, 469, L33

Lewin, W. H. G., McClintock, J. E., Ryckman, S. G., \& Smith, W. B. 1971, ApJ, 166, L69

Lutovinov, A. A., \& Tsygankov, S. S. 2009, Astronomy Letters, 35, 433

Makino, F., Leahy, D. A., \& Kawai, N. 1985, Space Science Reviews, 40, 421

Mukherjee, U., \& Paul, B. 2004, A\&A, 427, 567

Nagase, F. 1989, PASJ, 41, 1

Parmar, A. N., White, N. E., \& Stella, L. 1989, ApJ, 338, 373

Pellizzoni, A., et al. 2009, ApJ, 691, 1618

Pestalozzi, M., Torkelsson, U., Hobbs, G., \& López-Sánchez, Á. R. 2009, A\&A, 506, L21

Pravdo, S. H., Day, C. S. R., Angelini, L., Harmon, B. A., Yoshida, A., \& Saraswat, P. 1995, ApJ, 454, 872

Pravdo, S. H., \& Ghosh, P. 2001, ApJ, 554, 383

Saraswat, P., et al. 1996, ApJ, 463, 726

Sato, N., Nagase, F., Kawai, N., Kelley, R. L., Rappaport, S., \& White, N. E. 1986, ApJ, 304, 241

Swank, J. H., Becker, R. H., Boldt, E. A., Holt, S. S., Pravdo, S. H.,

Rothschild, R. E., \& Serlemitsos, P. J. 1976, ApJ, 209, L57

Tavani, M., et al. 2009, A\&A, 502, 995

Taylor, J. H., \& Weisberg, J. M. 1989, ApJ, 345, 434

White, N. E., Mason, K. O., Huckle, H. E., Charles, P. A., \& Sanford, P. W. 1976, ApJ, 209, L119

White, N. E., Mason, K. O., \& Sanford, P. W. 1978, MNRAS, 184, 67P 